Research Paper

\title{
PLCB4 copy gain and PLCB4 overexpression in primary gastrointestinal stromal tumors: Integrative characterization of a lipid-catabolizing enzyme associated with worse disease- free survival
}

\author{
Chien-Feng Li, ${ }^{1,3,8}$, Ting-Ting Liu ${ }^{4}$, I-Chieh Chuang ${ }^{4,8}$, Yen-Yang Chen ${ }^{5}$, Fu-Min \\ Fang $^{6}$, Ti-Chun Chan ${ }^{1}$, Wan-Shan Li ${ }^{7,8}$, Hsuan-Ying Huang ${ }^{4,8}$ \\ ${ }^{1}$ Department of Pathology, Chi-Mei Medical Center, Tainan, Taiwan \\ ${ }^{2}$ National Institute of Cancer Research, National Health Research Institutes, Tainan, Taiwan \\ ${ }^{3}$ Department of Biotechnology, Southern Taiwan University of Science and Technology, Tainan, Taiwan \\ ${ }^{4}$ Department of Pathology, Kaohsiung Chang Gung Memorial Hospital and Chang Gung University College of Medicine, \\ Kaohsiung, Taiwan \\ ${ }^{5}$ Division of Oncology, Department of Internal Medicine, Kaohsiung Chang Gung Memorial Hospital and Chang Gung University \\ College of Medicine, Kaohsiung, Taiwan \\ ${ }^{6}$ Department of Radiation Oncology, Kaohsiung Chang Gung Memorial Hospital and Chang Gung University College of \\ Medicine, Kaohsiung, Taiwan \\ ${ }^{7}$ Department of Pathology, Kaohsiung Medical University Hospital, Kaohsiung Medical University, Kaohsiung, Taiwan \\ ${ }^{8}$ Bone and Soft Tissue Study Group, Taiwan Society of Pathology, Taiwan \\ Correspondence to: Hsuan-Ying Huang, email: cfli.hyhuang@gmail.com \\ Keywords: metabolism, lipid catabolism, PLCB4, transcriptome, GIST \\ Abbreviations: GIST, gastrointestinal stromal tumor; NIH, National Institute of Health; NCCN, National Comprehensive Cancer \\ Network; PLCB4, phospholipase C-B4. \\ Received: October 09, $2016 \quad$ Accepted: December 08, $2016 \quad$ Published: February 13, 2017
}

\section{ABSTRACT}

To explore the implications of lipid catabolism-associated genes in gastrointestinal stromal tumors, we reappraised transcriptomic and genomic datasets and identified copygained and differentially upregulated PLCB4 gene associated with tumor progression. On full sections, PLCB4 mRNA abundance and PLCB4 immunoexpression were validated in 70 cases. On tissue microarrays, PLCB4 gene copies and PLCB4 immunoexpression were both informative in 350 cases with KIT/PDGFRA/BRAF genotypes noted in 213. In GIST48 cell line, we stably silenced PLCB4 and YAP1 to characterize their functional effects and regulatory link. Compared with normal tissue, PLCB4 mRNA abundance significantly increased across tumors of various risk levels $(p<0.001)$, and was strongly correlated with immunoexpression level $(p<0.001, r=0.468)$. Including polysomy $(12.6 \%)$ and amplification (17.4\%), PLCB4 copy gain was detected in $105(30 \%)$ cases and significantly more frequent $(p<0.001)$ in cases exhibiting higher PLCB4 immunoexpression (82/175). Copy gain and protein overexpression were modestly associated with unfavorable genotypes (both $\mathrm{p}<0.05$ ), strongly associated with increased size, mitosis, and risk levels defined by both the NIH and NCCN schemes (all $p<0.001$ ), and univariately predictive of shorter disease-free survival (both $p<0.0001$ ). In PLCB4-overexpressing cases, PLCB4 copy gain still predicted worse prognosis $(p<0.0001)$. In a multivariate comparison, both overexpression $(p=0.007$, hazard ratio: $2.454)$ and copy gain ( $p=0.031$, hazard ratio: 1.892) exhibited independent impact. In vitro, YAP1 increased PLCB4 mRNA and protein expression, and both molecules significantly promoted cell proliferation. Being driven by copy gain or YAP1, PLCB4 is a novel overexpressed enzyme regulating lipid catabolism that promotes cell proliferation and independently confers a worse prognosis. 


\section{INTRODUCTION}

The majority of gastrointestinal stromal tumors (GISTs) harbor mutually exclusive KIT or PDGFRA mutations, which drive tumor inception and dictate treatment response to imatinib [1]. Although the prognostic utility of NIH and NCCN risk schemes is effectual [2, $3]$, better characterization of molecular aberrations in tumor progression may refine prognostication of GISTs [4]. Clinical trials recently demonstrated the benefit of adjuvant imatinib therapy for moderate-risk and highrisk GISTs [5], which renders counseling regarding outcomes imperative, particularly in cases harboring imatinib-sensitive KIT/PDGFRA genotypes. In GISTs, secondary drug resistance to imatinib or other kinasestargeting agents inevitably emerges following initial responses [6], a challenge necessitating identification of novel non-kinase targets. It is desirable to characterize molecular aberrations of other signaling pathways, such as deregulated metabolism-associated enzymes that provide cellular energy and building blocks to sustain proliferation and metastasis of cancer cells [7] but remain largely unexplored in GISTs.

In this study, elucidation of lipid metabolismassociated enzymes was hypothesized to facilitate risk assessment. We began reappraising the transcriptomic datasets to search for metabolic driver genes involving the lipid catabolic process, which exhibited differential upregulation in high-risk and metastasizing GISTs at diagnosis with concordant copy number alterations (CNAs) in genomic profiling. This data-mining approach identified $P L C B 4$ as a top-ranking upregulated candidate with non-random copy gain in aggressive tumors. To the full extent of genetic, transcriptional, and translational characterization, we independently validated the clinical relevance of PLCB4 copy gain and PLCß4 overexpression in GISTs. PLCB4 encodes the $B 4$ isoform of phosphoinositide-specific phospholipase C (PLC) isoenzymes, a superfamily orchestrating the metabolism of inositol lipids [8]. The PLCß4 immunoexpression level had strong correlations with DNA copies and mRNA abundance. These genetic and expressive alterations exhibited robust associations with adverse clincopathological factors, unfavorable genotypes, and worse outcomes. Through polysomy or amplification, PLCB4 copy gain represents one mechanism driving PLCß4 ovexpression and conferring biological aggressiveness, with strong negative prognostic impact in both the entire cohort and the PLCB4-overexpressing sub-cohort. Using RNA interference in a PLCß4expressing GIST cell model, we functionally validated the pro-proliferative oncogenic attribute of PLCB4 and its expression upregulated by increased YAP1, hence providing insights into alternative non-amplified mechanism(s) that may drive PLCB 4 expression.

\section{RESULTS}

\section{Differential $P L C B 4$ mRNA upregulation and copy gain in aggressive GISTs}

Focusing on 142 probes covering 77 genes regulating lipid catabolic process, unsupervised hierarchic clustering was performed for two transcriptomic datasets of GISTs and crudely separated the samples into two clusters. These comprised 7 and 9 genes differentially expressed between high-risk and non-high-risk samples in GSE8167 (Figure 1A, Supplementary Table 1) and between localized and metastasizing samples in GSE20708 (Figure 1B, Supplementary Table 2), respectively. Notably, PLCB4 represented the top-ranking upregulated candidate common to both datasets, exhibiting strong associations with high-risk category and metastasis (both $\mathrm{p} \leq 0.0001$ ) and remarkable expression fold changes ( $\log _{2}$ ratios: 1.1827-2.4364). In the genomic dataset (GSE21185), the PLCB4 copy number was non-randomly gained in $20 \%$ of samples (Figure 1C). In this context, we selected PLCB4 to validate its clinical relevance in two independent tumor cohorts.

\section{PLCB 4 mRNA abundance associated with risk categories and immunoexpression}

To validate the findings of transcriptomic reappraisals by Quantigene PLCB4 mRNA quantification, we preselected cases having concordant assignment of high-risk versus non-high-risk category using both NCCN and NIH criteria $[2,3]$. The detection of PLCB4 mRNA abundance was informative in 10 normal tissues and 70 primary GISTs. Another 16 tumors could not be analyzed because of degraded nucleic acids. These 70 informative GISTs, 49 gastric and 21 intestinal, were classified as 20 high-risk and 50 non-high-risk cases (Supplementary Table 3). Compared with normal tissues, PLCB4 mRNA abundance was significantly higher in GISTs as a whole $(\mathrm{p}<0.001$, Figure 1D) and also increased from normal tissue over the non-high-risk group ( $\mathrm{p}=0.007)$ to highrisk GISTs $(\mathrm{p}=0.008)$, indicating its implication in tumor progression. Notably, mRNA and protein levels of PLCB4 were strongly and positively associated with each other $(\mathrm{p}<0.001, \mathrm{r}=0.468$, Figure 1E).

\section{PLCB 4 copy gain and PLCB4 overexpression associated with each other, adverse clinicopathological factors, and unfavorable RTK genotypes}

To characterize the clinical relevance of PLCB4, a TMA validation set was employed to evaluate gene copies and immunoexpression, yielding 350 GISTs informative for both variables and follow-up data (Tables 1,2). These 
comprised 88 none- or very low-risk, 100 low-risk, 65 moderate-risk, and 97 high-risk cases based on NCCN guidelines, corresponding to 127 very low/low-risk, 110 intermediate-risk, and 113 high-risk cases according to NIH risk scheme (Supplementary Table 4). Among 213 GISTs previously analyzed for KIT and PDGFRA mutations, 22 cases harboring normal KIT/PDGFRA genotypes were confirmed to retain expression of SDHA and SDHB (Supplementary Figure 1A) and wild-type $B R A F$ gene (Supplementary Figure $1 \mathrm{~B}$ ). The median value of averaged PLCB4 immunohistochemical H-scores was 245 (range, 100-390) and used to dichotomize overexpressed and under-expressed groups $(n=175$ each) (Figure 2A). The overexpressed group strongly correlated with increased PLCB4 copies (Figure 2B) detected in $105(30 \%)$ cases $(\mathrm{p}<0.001)$, including polysomy in $44(12.6 \%)$ and amplification in 61(17.4\%). The vast majority (59/61) of amplified cases exhibited PLCß4 overexpression, which was observed only in a half $(23 / 44)$ of polysomic cases. However, more than a half $(53.1 \%$, 93/175) of PLCß4-overexpressing tumors did not display PLCB4 copy gain by FISH, suggesting that alternative mechanism(s) may drive PLCß4 overexpression in GISTs.
Increased $P L C B 4$ copies and PLC 34 overexpression were modestly associated with unfavorable genotypes (both $\mathrm{p}<0.05$ ) and strongly related to increased size, mitosis, and risk levels defined by both NIH and NCCN schemes (all $\mathrm{p}<0.001$, Table 1). However, non-gastric locations and presence of epithelioid histology were only associated with overexpression $(p=0.022)$ and copy gain $(p<0.001)$, respectively.

\section{Copy gain-driven PLCß4 overexpression was predictive of worst prognosis}

At the univariate level, PLCB4 copy gain and PLCB4 overexpression were robustly predictive of shorter DFS (Figure 3A, 3B, Table 2, Supplementary Table 4, both $\mathrm{p}<0.0001)$ for the entire cohort. Moreover, GISTs exhibiting both copy gain and overexpression fared significantly worse than the counterparts featuring either one or none of both aberrations (Figure $3 \mathrm{C}, \mathrm{p}<0.0001$ ), while the latter two groups were not prognostically different. Notably, difference in $P L C B$ gene status also exhibited strong prognostic impact (Figure 3D, $\mathrm{p}<0.0001)$ wherein the most unfavorable DFS predictor


Figure 1: Transcriptomic and genomic reappraisals identify $P L C B 4$ as the top-ranking lipid catabolism-regulating gene associated with aggressive GISTs. A, B. Heatmaps of unsupervised hierarchical clustering analysis for differentially expressed genes between non-high-risk and high-risk samples (A, GSE8167) and between localized and metastasizing GISTs (B, GSE20708) at diagnosis. In both comparisons, two each crudely segregated clusters were identified, which comprised more high-risk (yellow bars) and metastasizing (red bars) GITSs in the upper left quadrants, respectively. Listed on the right of individual heatmaps were differentially upregulated (red) and downregulated (green) genes involving the lipid catabolic process (GO: 0006629). C. Analysis of genomic profiling dataset in the public domain (GSE21185) reveals an amplicon at 20p12.2-12.3 harboring PLCB4 gene, exhibiting copy gain in 20\% (4 out of 20) GISTS. D, E. In the Quantigene assay (D), PLCB4 mRNA was validated to be differentially upregulated across GISTs of various risk levels (informative $\mathrm{n}=70$ ) as compared with the normal tissues. PLCB4 mRNA also exhibits significant risk level-associated increment, implying its role in tumor progression. In the same set of 70 GISTs, the scattered plot (E) showed a strong correlation between the PLCB4 mRNA level on the $X$ axis and H-scores of PLCB4 immunoexpression on the $Y$ axis (see representative images in Figure 2A). 
Table 1: Associations of PLCB4 expression and gene dosage with various clinicopathological parameters in 350 GIST patients

\begin{tabular}{|c|c|c|c|c|c|c|}
\hline & \multicolumn{2}{|c|}{ PLCB4 Expression } & \multirow{2}{*}{ p-value } & \multicolumn{2}{|c|}{$P L C B 4$ Gene Dosage } & \multirow{2}{*}{ p-value } \\
\hline & Low Exp. & High Exp. & & No gain & Polysomy \& Amp. & \\
\hline Sex & & & 0.748 & & & 0.363 \\
\hline Male & 87 & 90 & & 125 & 57 & \\
\hline Female & 88 & 85 & & 120 & 48 & \\
\hline Age (years) & $58.9 \pm 13.70$ & $60.9 \pm 11.70$ & 0.137 & $59.9 \pm 13.14$ & $59.7 \pm 11.90$ & 0.900 \\
\hline Location & & & $0.022 *$ & & & 0.082 \\
\hline Gastric & 116 & 95 & & 155 & 56 & \\
\hline Non-gastric & 59 & 80 & & 90 & 49 & \\
\hline Histologic Type & & & 0.802 & & & $<0.001 *$ \\
\hline Spindle & 134 & 132 & & 200 & 66 & \\
\hline Epithelioid \& Mixed & 41 & 43 & & 45 & 39 & \\
\hline Tumour Size $(\mathrm{cm})^{\&}$ & $5.4 \pm 3.85$ & $7.4 \pm 4.35$ & $<0.001 *$ & $5.4 \pm 3.47$ & $8.6 \pm 4.93$ & $<0.001 *$ \\
\hline Mitotic Count (50HPFs) ${ }^{\&}$ & $6.5 \pm 18.94$ & $12.0 \pm 26.95$ & $0.001 *$ & $5.3 \pm 12.24$ & $18.3 \pm 37.01$ & $<0.001 *$ \\
\hline NIH Risk & & & $<0.001 *$ & & & $<0.001 *$ \\
\hline Low/Very low & 85 & 42 & & 116 & 11 & \\
\hline Intermediate & 54 & 56 & & 81 & 29 & \\
\hline High & 36 & 77 & & 48 & 65 & \\
\hline NCCN Guideline & & & $<0.001 *$ & & & $<0.001 *$ \\
\hline None/Very low & 66 & 22 & & 82 & 6 & \\
\hline Low & 50 & 50 & & 81 & 19 & \\
\hline Moderate & 32 & 33 & & 40 & 25 & \\
\hline High & 27 & 70 & & 42 & 55 & \\
\hline Mutation Type & & & $0.033 *$ & & & $0.023 *$ \\
\hline Favorable Type & 54 & 52 & & 78 & 28 & \\
\hline Unfavorable Type & 39 & 68 & & 63 & 44 & \\
\hline PLCB4 Expression & & & & & & $<0.001 *$ \\
\hline Low Exp. & & & & 152 & $23\left(21^{\#}, 2^{\$}\right)$ & \\
\hline High Exp. & & & & 93 & $82\left(23^{\#}, 59^{\$}\right)$ & \\
\hline
\end{tabular}

*: Statistically significant, \&: Wilcoxon rank-sum test, \#, \$: numbers of polysomic and amplified cases, respectively.

was amplification ( $p<0.0001$, vs. polysomy), followed by polysomy ( $\mathrm{p}=0.0216$, vs. normal status). Even if only PLCß4-overexpressing GISTs were considered, DFS was still negatively related to $P L C B 4$ copy gain (Figure $3 \mathrm{E}, \mathrm{p}<0.0001)$ and increased $P L C B$ gene copy numbers (Figure $3 \mathrm{~F}, \mathrm{p}<0.0001$ ), with significant differences in amplification vs. polysomy $(\mathrm{p}=0.0290)$ and in polysomy vs. normal status $(\mathrm{p}=0.0030)$. These clearly indicated the clinical relevance of determining the actual gene status in PLCß4-overexpressing cases.

Regarding the factor of risk levels, we separately evaluated the prognostic impact of either NCCN guidelines or NIH scheme in two multivariate regression models. When NCCN guidelines were incorporated (Table 2$)$, both PLCB4 copy gain $(\mathrm{p}=0.031$, hazard ratio: 1.892) and PLCß4 overexpression $(\mathrm{p}=0.007$, hazard 
Table 2: Univariate and multivariate analyses for disease-free survival according to PLCB4 gene status, PLCB4 expression status, NCCN guidelines, and other prognostic factors

\begin{tabular}{|c|c|c|c|c|c|c|}
\hline \multirow{2}{*}{ Parameter } & \multicolumn{3}{|c|}{ Univariate analysis } & \multicolumn{3}{|c|}{ Multivariate analysis } \\
\hline & No. Case & No. Event & p-value & HR & $95 \% \mathrm{CI}$ & p-value \\
\hline Sex & & & 0.4667 & & & \\
\hline Male & 177 & 43 & & & & \\
\hline Female & 173 & 44 & & & & \\
\hline Age (years) & & & 0.0584 & & & \\
\hline$<70$ & 259 & 59 & & & & \\
\hline$>=70$ & 91 & 28 & & & & \\
\hline Location & & & $0.0023 *$ & & & 0.385 \\
\hline Gastric & 211 & 40 & & 1 & - & \\
\hline Non-gastric & 139 & 47 & & 0.798 & $0.480-1.328$ & \\
\hline Histologic Type & & & $<0.0001 *$ & & & $0.001 *$ \\
\hline Spindle & 266 & 51 & & 1 & - & \\
\hline Mixed/Epithelioid & 84 & 36 & & 2.364 & $1.408-3.971$ & \\
\hline Tumour Size (cm) ${ }^{\#}$ & & & $<0.0001 *$ & & & \\
\hline$=<5 \mathrm{~cm}$ & 161 & 16 & & & & \\
\hline$>5 ;=<10 \mathrm{~cm}$ & 131 & 38 & & & & \\
\hline$>10 \mathrm{~cm}$ & 58 & 33 & & & & \\
\hline Mitotic Count (50HPFs) & & & $<0.0001 *$ & & & \\
\hline $0-5$ & 249 & 33 & & & & \\
\hline $6-10$ & 43 & 14 & & & & \\
\hline$>10$ & 58 & 40 & & & & \\
\hline NCCN Guideline & & & $<0.0001^{*}$ & & & $<0.001 *$ \\
\hline None/Very low & 88 & 3 & & 1 & - & \\
\hline Low & 100 & 10 & & 2.461 & $0.519-11.675$ & \\
\hline Moderate & 65 & 15 & & 2.566 & $0.541-12.173$ & \\
\hline High & 97 & 59 & & 8.408 & $1.913-36.963$ & \\
\hline Mutation Type & & & $0.0005^{*}$ & & & 0.091 \\
\hline Favorable type & 106 & 22 & & 1 & - & \\
\hline Unfavorable type & 107 & 45 & & 1.584 & $0.929-2.701$ & \\
\hline PLCB4 expression & & & $<0.0001 *$ & & & $0.007 *$ \\
\hline Low Expressed & 175 & 20 & & 1 & - & \\
\hline High Expression & 175 & 67 & & 2.454 & $1.274-4.730$ & \\
\hline$P L C B 4$ gene status & & & $<0.0001 *$ & & & $0.031 *$ \\
\hline No gain & 245 & 31 & & 1 & - & \\
\hline Polysomy \& Amp. & 105 & 56 & & 1.892 & $1.062-3.370$ & \\
\hline
\end{tabular}

\#, Tumour size and mitotic activity were not introduced in multivariate analysis, since these two parameters were component factors of NCCN risk scheme; *, Statistically significant. HR, hazard ratio. 
A
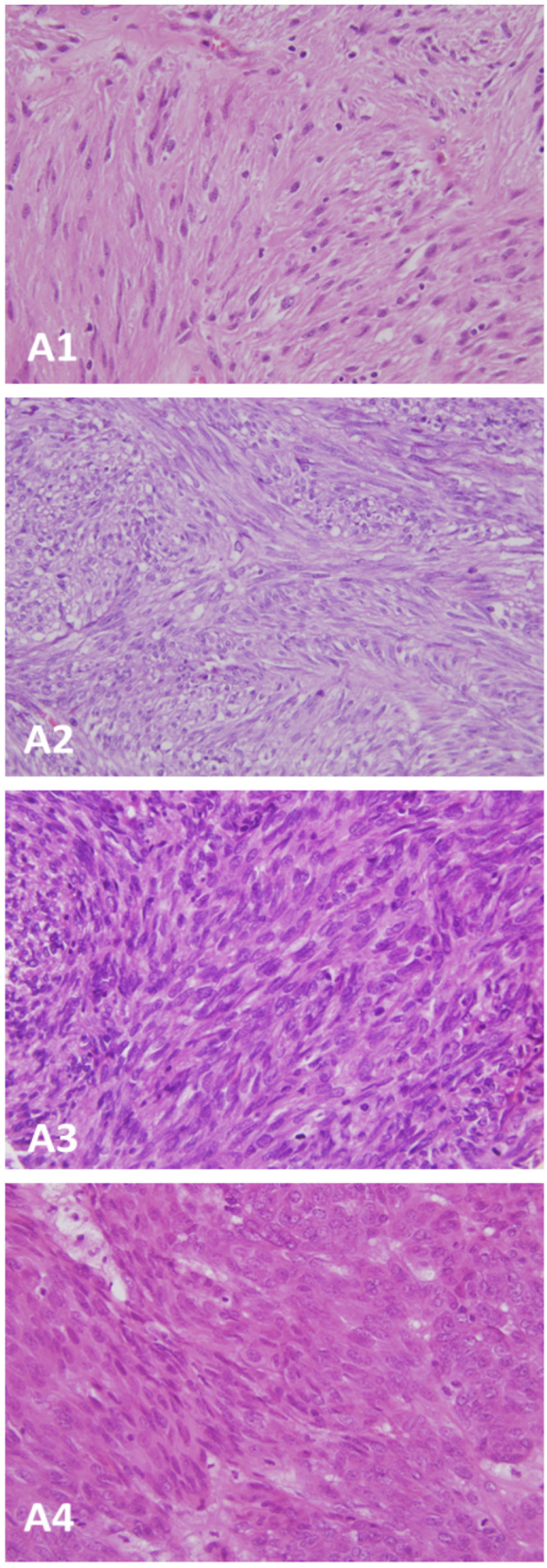


B


Figure 2: Independent validation for clinical implications of PLCB4 overexpression and PLCB4 copy gain on tissue microarrays. A. In one each representative GISTs classified as very low- $\left(A_{1}\right)$, low- $\left(A_{2}\right)$, intermediate- $\left(A_{3}\right)$, and high-risk $\left(A_{4}\right)$ levels, tumor cells exhibit no $\left(A_{5}\right)$, weak $\left(A_{6}\right)$, moderate $\left(A_{7}\right)$ to diffuse strong $\left(A_{8}\right)$ cytoplasmic reactivity to PLCB4, respectively. B. Using the probe directed against the centromeric sequence (green) as the reference, a locus-specific FISH probe targeting PLCB4 gene (red) demonstrated normal status $\left(\mathrm{B}_{1}\right)$, polysomy $\left(\mathrm{B}_{2}\right)$, and amplification $\left(\mathrm{B}_{3}\right)$ in one each representative GIST. 
ratio: 2.454) remained independently prognostic of worse outcomes, together with higher NCCN risk levels and presence of epithelioid histology (both $\mathrm{p}<0.001$ ). However, unfavorable genotypes only exhibited a trend toward marginal significance. When NIH scheme, rather than NCCN criteria, was considered, only PLC 34 protein overexpression remained prognostically significant $(p=0.001$, Supplementary Table 4$)$, together with higher $\mathrm{NIH}$ risk levels and presence of epithelioid histology (both $\mathrm{p}<0.001)$. However, PLCB4 copy gain and unfavorable genotypes were not prognostically independent.

\section{Pro-proliferative function of PLCB4 linked to the upregulation by YAP1}

As stated above, PCLB4 copy gain and PLCB4 overexpression both exhibited the strong prognostic negative impact on GISTs with discrepancy in their frequencies of aberrations. Accordingly, we used a PLCB4-expressing GIST48 cell line to explore the oncogenic function of PLCB4 in GISTs and its regulatory link to YAP1 as other possible non-amplified mechanism to increase expression in light of the recent discovery of involvement of PLCB4 in YAP1-active mesothelioma cell proliferation [9]. RNA interference was employed, and two shPLCB4 clones achieved stable silencing as validated by both quantitative reversetranscriptase polymerase chain reaction (RT-PCR) and western blotting assays (Figure 4A, 4B). Compared with shLacZ, both shPLCB4 clones significantly decreased proliferative GIST48 cells from $48 \mathrm{~h}$ or $72 \mathrm{~h}$ onward (Figure 4C), hence confirming the proliferationpromoting attribute of PLCß4 in GISTs. Further, we stably knocked down YAP1 expression and observed remarkably decreased levels of mRNA and protein (Figure 5A, 5B), which significantly attenuated the expression of PLC 34 protein and PCLB4 mRNA (Figure $5 \mathrm{~B}, 5 \mathrm{C})$, indicating a positive regulatory effect of YAP1 on PCLB4 expression at the mRNA level. From Day 1 onward, stable YAP1 knockdown even more drastically decreased cell proliferation than shPLCB4 (Figure 5D), implying that PLC 34 may represent only one of the downstream effectors of YAP1 in promoting cell proliferation.
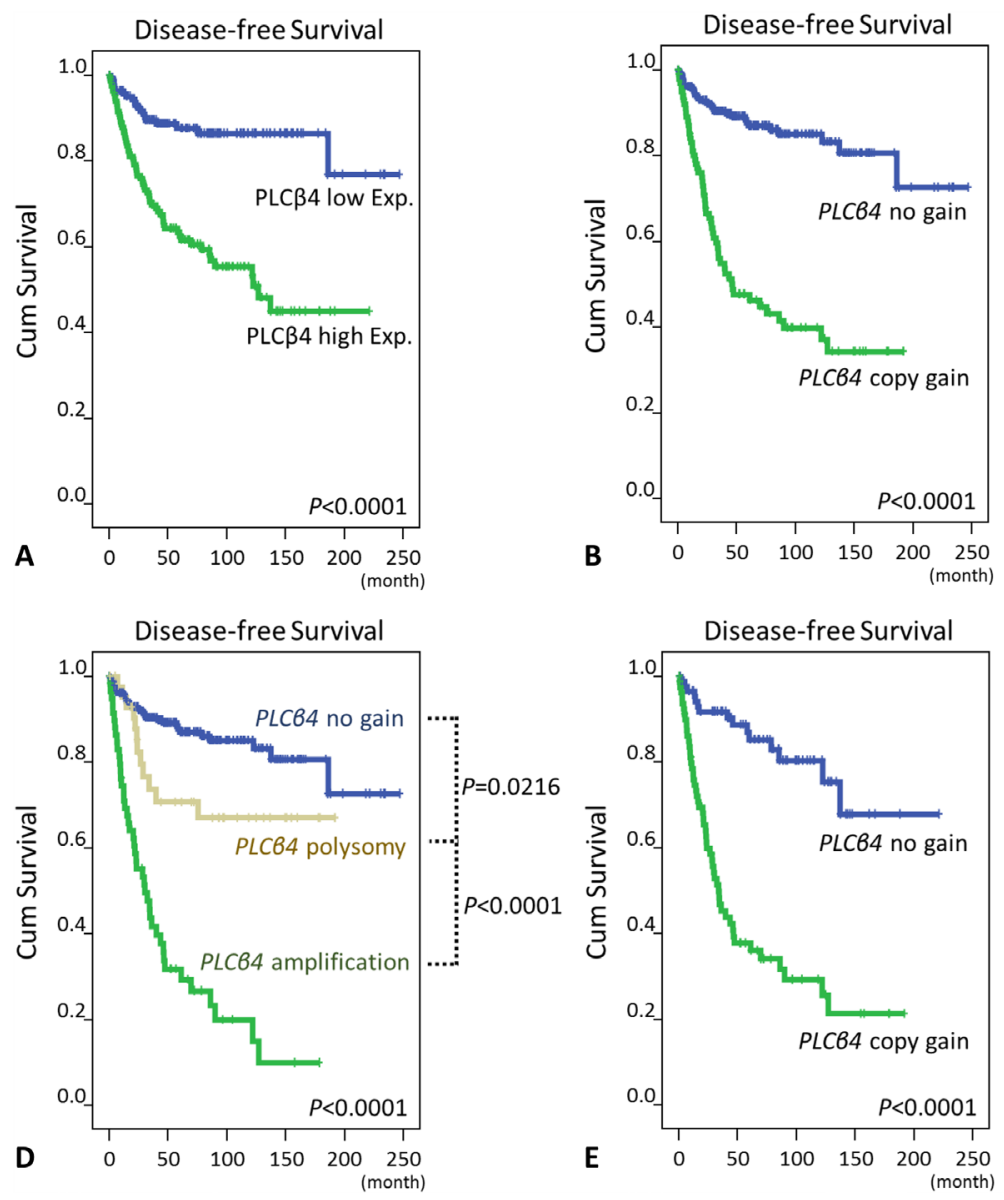
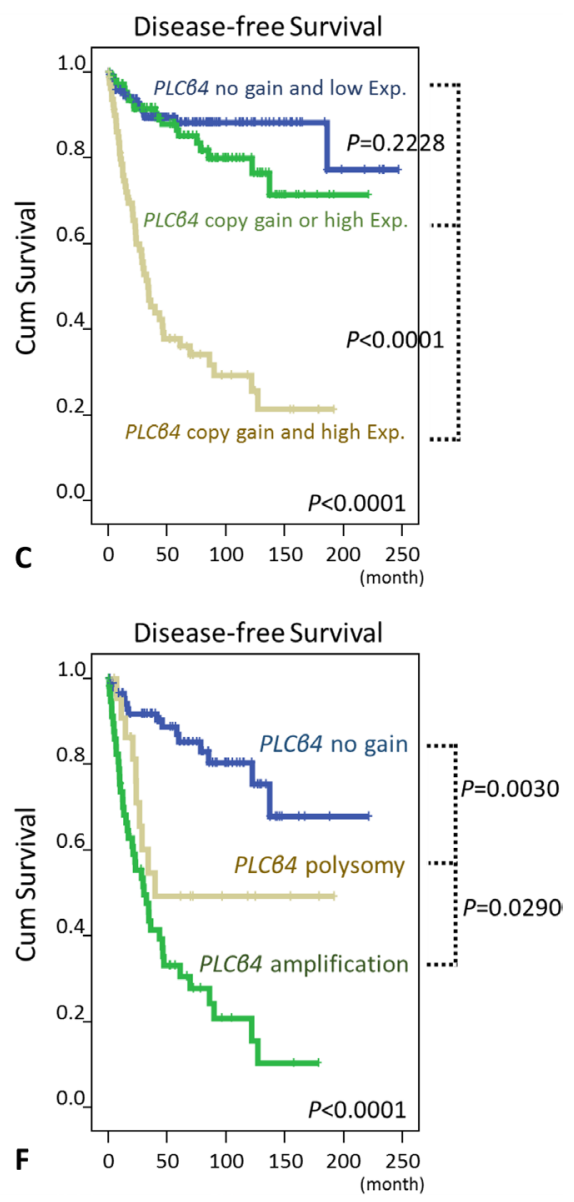

Figure 3: Kaplan-Meier analyses of univariate disease-free survival. In all 350 informative cases, survival curves were shown according to the presence or absence of PLC 34 overexpression A. and PLCB4 copy gain B., both aberrations combined versus neither or either of both $\mathbf{C}$., and the PLCB4 gene copy number status D. In 175 PLCß4-overexpressing cases, PLCB4 copy gain E. and the PLCB4 gene copy number status $\mathbf{F}$. also significantly distinguished GISTs with different outcomes. 


\section{DISCUSSION}

Among cancer-associated metabolic aberrations, accumulated CNAs attract increased attention for their dosage effects on the expression of lipid metabolismregulating genes $[10,11]$. Recently, an in silico study on glioblastomas indicated significant links between perturbed phosphoinositide metabolism and the CNAs of PLC isoenzymes-encoding genes, including amplified PLCB4 among others [11]. To coordinate signals modulating cellular processes, various PLCs isoenzymes regulate phospholipid turnover at the cellular membrane though hydrolyzing phosphoinositides (e.g., phosphatidylinositol4,5-diphospate) to generate diacylglycerol and inositol1,4,5-trisphosphate as secondary messengers $[12,13]$. Diacylglycerol mediates the activation of protein kinase $C$, while inositol-1,4,5-trisphosphate triggers calcium release from the intracellular reservoir $[12,13]$. Notably, PLC $\varepsilon$ and PLC $\gamma$ may confer diverse cancer-promoting functions through deranging the balance among phosphoinositidesassociated substrates, messengers, and signaling pathways $[12,13]$. In mesenchymal tumors, R707Q mutation



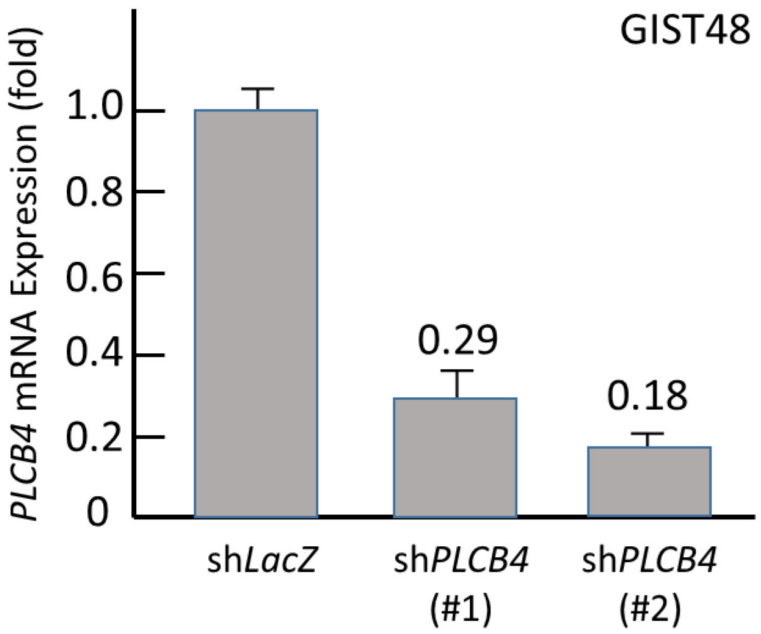

B GIST48

PLCB4


GAPDH

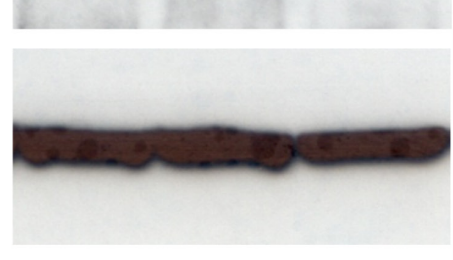

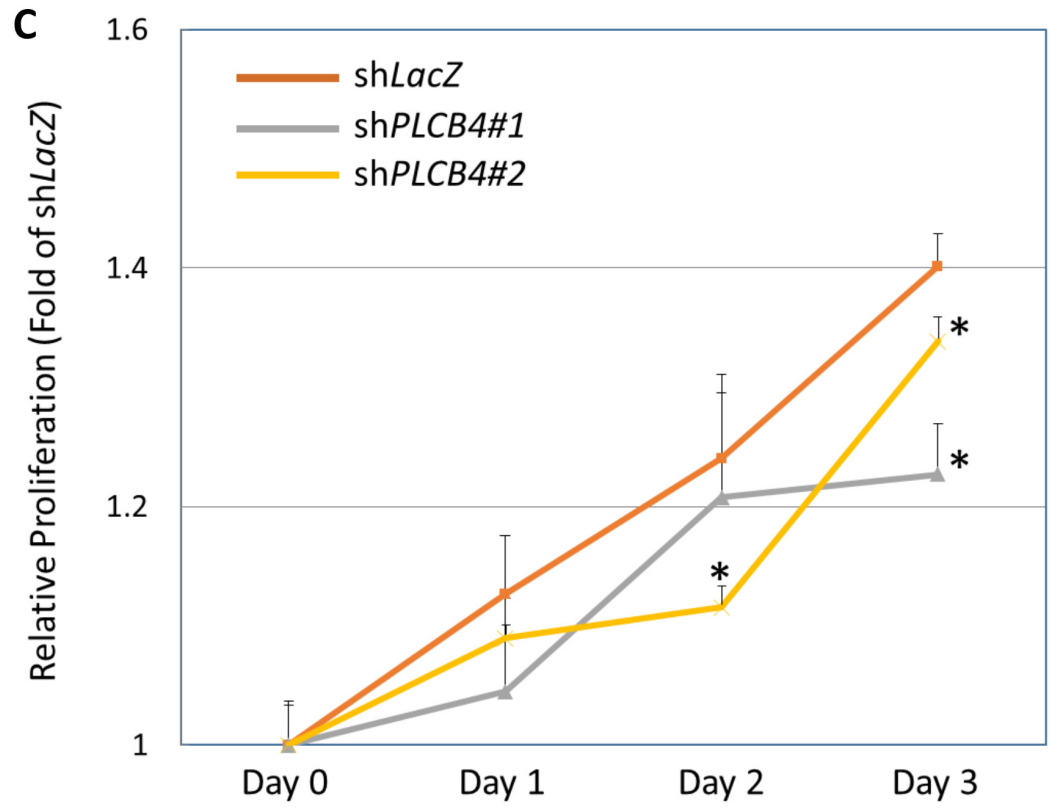

Figure 4: Stable RNA interference targeting $P L C B 4$ decreased cell proliferation. Compared with shLacZ, both clones of short hairpin RNAs against PLCB4, shPCLB4\#1 and shPLCB4\#2, remarkably decreased the mRNA and protein expression levels of PLCB4 in the PLCB4-expressing GIST48 cell line, as demonstrated by real-time RT-PCR A. and western blotting B. assays. When transduced with shPCLB4\#1 and shPLCB4\#2, the proliferation of GIST48 cells determined by the Brdu assay C. significantly declined from $72 \mathrm{~h}$ or $48 \mathrm{~h}$ onward, respectively. 
constitutively activates PLC $\gamma 1$ and promotes evasion of apoptosis, migration, and invasiveness in angiosarcomas [14].

Of PLCß isoforms, overexpressed PLCB2 in breast carcinomas exhibits oncogenic functions and poor prognostic impact through promoting $\mathrm{G} 2 / \mathrm{M}$ cell cycle progression and cell migration $[15,16]$. Being abundant in the cerebellum and retina [17], PLCß4 contributes to the development of the first and second pharyngeal arches in the embryo, and germline PLCB4 mutations occur in most patients with auriculocondylar syndrome, a rare craniofacial malformation manifesting mandibular deformity and characteristic "questionmark" ears [18]. However, the role of PLCB4 in cancer biology remains largely undefined, although a subset of uveal melanomas harbor a novel driver mutation at codon



C



Asp630 [8]. In human cancers, PLCB4 amplification has not been formally documented as a mechanism underlying overexpression, while it is provisionally catalogued in $20.6 \%$ of prostatic carcinomas exhibiting neuroendocrine phenotypes by the cBioPortal platform for cancer genomics [19].

As a proof of concept, we corroborated the clinical relevance of the increased dosage effect of PLCB4 gene in human neoplasms for the first time, highlighting the presumable conversion of copy gain into upregulated mRNA and overexpressed protein in an aggressive subset of GISTs. This reasoning is based on the strong positive associations of PLCB4 immunoexpression levels with mRNA abundance and gene copy status in two independent cohorts. Compared with non-tumoral tissues, the PLCB4 mRNA abundance was not only
B



D

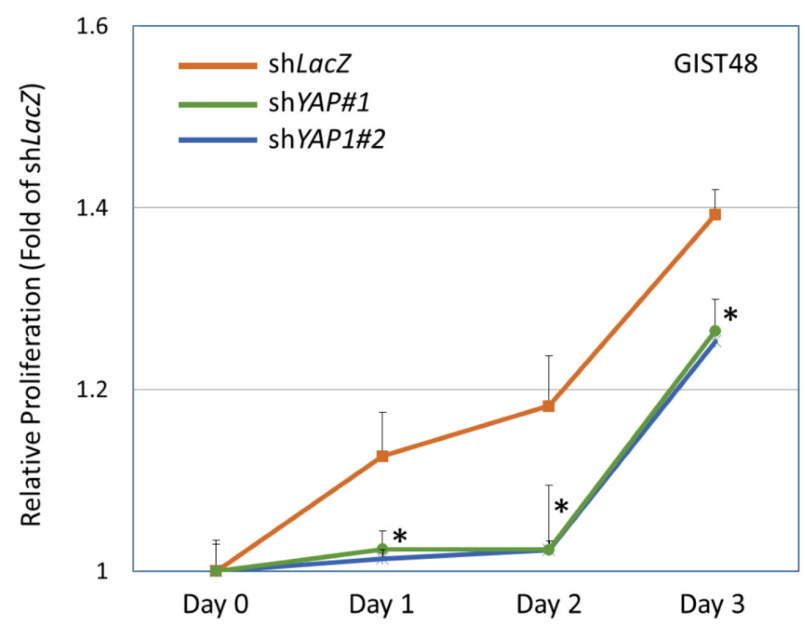

Figure 5: Stable RNA interference against YAP1 decreased PLCB4 expression and cell proliferation. Compared with shLacZ, short hairpin RNAs against YAP1, shYAP1\#1 and shYAP1\#2, effectively decreased the mRNA A. and protein B., (upper row) expression of YAP1 as determined by real-time RT-PCR and western blots, respectively. This genetic approach also caused significant decrease in the expression levels of PLCß4 protein (B, middle row) and PLCB4 mRNA C. Moreover, the cell proliferation of GIST48 cells transduced with either clone of shYAP1 D. exhibited remarkable decrease from $24 \mathrm{~h}$ onward, compared with shLacZ control. 
significantly higher in the whole group of tumors but also increased stepwise with risk increment. At the DNA and protein levels, PLCß4 overexpression and PLCB4 copy gain both robustly characterized GISTs featuring increased tumor size, mitosis, and higher risk levels defined by both NIH and NCCN schemes. These strong links indicated that the oncogenic role of PLCB4 in GISTs is, at least in part, attributable to copy gain, displaying polysomy or amplification as a manifestation of genetic instability. Intriguingly, we found modest associations of unfavorable KIT/PDGFRA/BRAF genotypes with both $P L C B 4$ copy gain and PLC 34 overexpression, whereas it remains indefinite whether these findings indeed connote biological links.

Gene amplification is one mechanism underlying overexpression of various oncoproteins, while increased gene dosage secondary to polysomy is not clearly established for its clinical and biological implications in cancers [20]. Even if PLCB4 overexpression and PLCB4 copy gain both predicted DFS, our results highlighted the clinical relevance of PLCB4 copy gain, being potentially distinctive from protein overexpression. In both the entire cohort and PLCß4-overexpressing GISTs, there were significantly different DFS rates between copynormal and polysomic cases and between polysomic and amplified cases. This finding resonated with the polysomy of chromosome 17 in breast carcinomas, which, to a lesser degree than $E R B B 2$ amplification, generally associates with clincopathological aggressiveness and worse prognosis [20]. Currently, there is lack of ample evidence to proclaim PLC 34 immunohistochemistry as a surrogate of PLCB4 FISH assay. The latter is desirable to determine the gene status in PLCß4-overexpressing GISTs for prognostic reference and needful for the prospective follow-up in the PLCB4-polysomic cases. In the multivariate model incorporating NCCN risk levels, we substantiated that both PLCB4 overexpression and PLCB4 copy gain were independent prognosticators, conceivably denoting that PLCB4 confers growth advantages and exasperates the progression of GISTs. However, PLCB4 copy gain was detected only approximately a half (47\%) of PLCß4-overexpressing tumors in our TMA cohort, indicating operation of alternative upregualtory mechanism(s). In this regard, we further demonstrated that PLCß4 contributed to cell proliferation in a PLCß4expressing GIST cell line, and increased expression of YAP1, also exhibiting a pro-proliferative attribute, could upregulate $P L C B 4$ expression likely at the mRNA level.

Conclusively, we have corroborated PLC 34 as an oncogenic lipid-catabolizing enzyme in primary imatinibnaïve GISTs, which can be driven by amplification- or polysomy-derived copy gain or upregulated by increased YAP1. Given their strong associations with the risk increment, copy gain is presumably converted into upregulated mRNA and overexpressed protein, hence defining an aggressive subset of GISTs independently predicted by $P L C B 4$ copy gain and protein overexpression for shorter DFS. Copy gain and protein overexpression are not fully equivalent in prognostic relevance, for which performance of PLCB4-specfic FISH assay is warranted to complement PLCB4 immunostaining in GISTs. Further investigations on deregulated PLCß4 caused by gene copies versus YAP1-mediated upregulation may better delineate the clinical implications of perturbed phosphoinositides metabolism and open an alternative therapeutic avenue for imatinib-resistant, high-risk GISTs.

\section{MATERIALS AND METHODS}

\section{Reappraisal of published genomic and transcriptomic datasets}

Transcriptomic datasets in Gene expression Omnibus were reappraised for imatinib-naïve gastric and intestinal GISTs with varying clinical and histological aggressiveness. The raw CEL files were $\log _{2}$-transformed and integrated using Nexus Expression 3 software (BioDiscovery Hawthorne, CA) to analyze probe sets associated with the lipid catabolic process in Gene Ontology (GO: 0006629). Unsupervised comparative analysis was performed to identify genes differentially upregulated in high-risk (GSE8167) and metastasizing (GSE20708) GISTs profiled by Affymetrix Microarray (HG-U133_Plus_2), compared with their non-high-risk and localized counterparts at diagnosis, respectively. In the genomic dataset (GSE21185) profiled by Agilent (G4412A) CGH Microarray, the somatic CNAs were profiled to evaluate the frequencies of concordant copy gain or deletion on chromosomes harboring differentially expressed genes in transcriptomic reappraisal and to depict the zoom-in view of chromosome 20p where PLCB4 is located. To delineate the breakpoints, gains and losses in significant CAN regions were defined as $\log _{2}$ ratios of $\geqq+0.20$ or $\leqq-0.20$, respectively. The ranking in the expression fold changes (requiring at least $\geqq 0.1$ fold in $\log _{2}$-transformed ratio), the power of statistical significance $(\mathrm{p} \leq 0.01$ by Student- $t$ test), and the concordance with DNA copy alterations in genomic profiling were considered for prioritizing genes for validation.

\section{Validation cohorts}

The institutional review boards of Chang Gung Hospital approved this study (102-3911B) under local ethical regulations. To validate the transcriptomic analysis, Quantigene measurement for PLCB4 mRNA abundance was performed on 86 formalin-fixed, primary GISTs, including 10 recent cases with adjacent non-tumoral tissue selected as the control. Of these, 70 informative cases were assessed for PLCß4 immunoexpression on full sections. Another independent cohort comprised 370 primary tumors resected before 2009, from which triplicate cores 
for each case had been previously assembled into tissue microarrays (TMA) [21]. TMA sections were recut for PLCB4-specfic fluorescent in situ hybridization (FISH) and PLCß4 immunohistochemistry, yielding 350 informative cases in both assays with 213 successfully genotypes for $K I T / P D G F R A$ as previously reported [21]. In both cohorts, all cases were imatinib-naïve before disease relapse and tabulated for clinicopathological characteristics listed in Table 1 and Supplementary Table 1.

\section{Quantigene branched-chain DNA assay}

This assay employs a sandwich nucleic acid hybridization to quantitate the mRNA abundance of housekeeping and target transcripts in tissue homogenates of formalin-fixed specimens [22]. Briefly, specific probes targeting PLCB4 transcript were customized for detection by QuantiGene Multiplex 2.0 assay system (Affymetrix/ Panomics). Oligonucleotides of the probe set were mixed with the lysed formalin-fixed tissues, and the mixture was added to a 96-well plate coated with capture probe oligonucleotides. Target RNA was captured and incubated overnight at $55^{\circ} \mathrm{C}$ with removal of unbound material using $300 \mu \mathrm{l}$ wash buffer for 3 runs, followed by hybridization of DNA amplifier molecules and three additional washes after incubation every time. The dioxetane alkaline phosphatase substrate Lumiphos Plus was added to the reaction wells for detection by Luminex 100 microplate luminometer (Luminex). The detected readout of PLCB4 mRNA abundance was further normalized by the expression level of reference GAPDH transcript.

\section{$P L C B 4$-specific FISH}

A bacterial artificial chromosome probe (RP11252K6, Invitrogen), spanning PLCB4 at 20p12.2, was labeled with SpectrumOrange. The Chromosome 20 Control probe targeting the centromeric region (\#041015, Empire Genomics) was labeled with Green 5-Fluorescein dUTP essentially following the previously described method [23]. PLCB4 copy number was assessed on 4- $\mu \mathrm{m}$ TMA sections by these locus-specific probes using a routine FISH protocol. The average numbers of red and green signals were determined by examining approximately 200 tumour cells in triplicate tissue cores for each specimen. Gene amplification was defined as a ratio of the gene probe signal to the control probe signal (i.e., red/green) exceeding 2.5. Polysomy was called when the average number of control green signals per nucleus was $\geqq 3$, with the red/green ratio being $\geqq 1$ and $<2.5$.

\section{Immunohistochemistry for PLCB4, SDHA, and SDHB}

Whole block and TMA sections were microwaveheated to retrieve tissue antigen and incubated with the primary antibody against PLCß4 (1:50; Abcam), followed by detection with ChemMate EnVision kit (DAKO) as our previous works [24, 25, 26]. Blind to molecular and survival data, one pathologist (T.T.L) independently assessed PLCß4 cytoplasmic expression using the method of H-score $[26,27]$, as defined by the equation, $\Sigma P_{i}(i$ $+1)$ where $i$ is the intensity of stained tumour cells $(0$ $3+)$ and $P i$ is the percentage of stained tumour cells ranging from $0 \%$ to $100 \%$. Regarding the TMA cohort, PLCß4 immunoexpression level was dichotomized into overexpressed and low-expressed groups, and the cutoff was defined as the median value of individual averaged triplicate $\mathrm{H}$-scores of 350 informative cases. To screen GISTs with potential defects in the succinate dehydrogenase (SDH) complex, 22 GISTs harboring a normal KIT/PDGFRA/BRAF genotype were evaluated for immunoexpression of SDHA (1:750; Abcam) and SDHB (1:200; Abcam) on full sections (Supplementary Figure 1A).

\section{Mutation analysis of $K I T, P D G F R A$, and $B R A F$ genes}

We had previously genotyped 213 GISTs through DNA extraction, PCR amplification, direct sequencing of KIT exon 11, and denatured high performance liquid chromatography screening for exons 9, 13, and 17 of the KIT gene and exons 12 and 18 of the PDGFRA gene with confirmatory sequencing [21]. Following reported thermal conditions and primer pairs [28], BRAF gene was only sequenced for 22 GISTs retaining the wild-type $K I T$ and $P D G F R A$ genes, given that the concurrence of $B R A F$ mutation with either KIT or PDGFRA mutation is extraordinary rare, if any, in GISTs [29, 30]. In fact, all these 22 cases revealed no mutation in the hotspot exon 15 that encompasses codon Val600 (Supplementary Figure 1A).

\section{Cell culture}

GIST48 cell line, a kind gift from Professor Jonathan Fletcher, is known to harbor a primary homozygous V560D mutation in KIT exon 11 and acquire a secondary heterozygous D820A mutation in KIT exon17 following imatinib therapy. As previously described [21], GIST48 cells were cultured in IMDM (Invitrogen) supplemented with $15 \%$ fetal bovine serum (FBS), $100 \mathrm{U} / \mathrm{ml}$ penicillin/ streptomycin, and $4 \mathrm{mM} \mathrm{L}$-glutamine (Invitrogen) at $37^{\circ} \mathrm{C}$ in $5 \% \mathrm{CO}_{2}$.

\section{RNA interference}

To establish stably silenced clones of GIST48 cell line with the short-hairpin RNAs against endogenously expressed $P L C B 4$ or $Y A P 1$, the lentiviral vectors were obtained from Taiwan National RNAi Core Facility, including pLKO.1-shLacZ (TRCN0000072223: 5'-TGT TCGCATTAT CCGAACCAT-3'), pLKO.1-shPLCB4 
(TRCN0000007012: 5'-CGCTGACATCAGATCACA AAT-3' designated as shPLCB4\#1; TRCN0000007013: 5'-CCTGAGATCAATCATACACAA-3' as shPLCB4\#2) and pLKO.1-shYAP1 (TRCN0000107268: 5'-GACCAAT AGCTCAGATCCTTT-3' as shYAPl\#1; TRCN0000107 269: 5'-CGACCAATAGCTCAGATCCTT-3' as shYAP1\#2). Viruses were produced by transfecting HEK293 cells with the vectors above using Lipofectamine 2000. For viral infection, $3 \times 10^{6}$ GIST48 cells were incubated with $8 \mathrm{ml}$ lentivirus in the presence of polybrene, followed by puromycin selection for stable clones of lentivirus-transduced cells.

\section{Quantification of transcripts of PLCB4 and YAP1}

RNeasy Mini kit (Qiagen, Valencia, CA) was used to extract total RNAs from stable clones of GIST cell lines with lentiviral vectors bearing shPLCB4, shYAP1, or shLacZ. RNAs were further reverse-transcribed using SuperScript ${ }^{\mathrm{TM}}$ III First-Strand Synthesis System (Invitrogen, Carlsbad, CA) according to the manufacturers' instructions. Real-time RT-PCR was performed using an ABI StepOnePlus ${ }^{\mathrm{TM}}$ System to quantify the expression levels of PLCB4 and YAP1 transcripts using pre-designed TaqMan assay reagents (Hs00168656_ml for $P L C B 4$, Hs00902712_g1 for $Y A P 1$, and POLR2A [a.k.a, RNA polymerase polypeptide A] Hs01108291_ml, Applied Biosystems, Foster City, CA). The obtained data were normalized by the expression of POLR $2 A$ housekeeping transcript. The relative expression fold of $P L C B 4$ or $Y A P 1$ mRNA was then given by $2^{-\Delta \Delta \mathrm{Cp}}$, where $\Delta \Delta \mathrm{C}_{\mathrm{T}}=$ $\Delta \mathrm{C}_{\mathrm{T}}\left({ }_{\text {shPLCB4 or shYAPI }}\right)-\Delta \mathrm{C}_{\mathrm{T}}\left({ }_{\text {sh } L a c Z}\right), \Delta \mathrm{C}_{\mathrm{T}}$ represented the $\mathrm{C}_{\mathrm{T}}$ of $P L C B 4$ or $Y A P 1$ subtracted from the $\mathrm{C}_{\mathrm{T}}$ of POLR $2 A$.

\section{Western blots}

The western blotting assay was performed to evaluate the endogenous PLCß4 expression and the efficiency and consequent alteration of silencing PCLB4 and YAP1 in GIST48 cells. Cell lysates containing 25 $\mu \mathrm{g}$ protein were separated by $4-12 \%$ gradient NuPAGE gel (Invitrogen), transferred onto PVDF membranes (Amersham), and probed with antibodies against PLCß4 (1:1000, BD Biosciences), YAP1 (1:10000, abcam), or GADPH (1;3000, Chemicon). After incubation with the secondary antibody, proteins were visualized by the chemiluminescence system (Amersham).

\section{Bromodeoxyuridine (BrdU) assay to assess DNA synthesis}

DNA synthesis was assessed using an enzymelinked immunosorbent assay-based and colorimetric BrdU assay (Roche Diagnostics) as previously described [21]. GIST48 cells transduced with shPLCB4, shYAP1, or shLacZ control were plated into a 96-well plate at density of 3000 cells per well, and DNA synthesis was evaluated at 24,48 , and $72 \mathrm{~h}$. The absorbance of the samples was measured using an ELISA reader (Promega) at $450 \mathrm{~nm}$, with the absorbance at $690 \mathrm{~nm}$ as reference.

\section{Statistical analysis}

For the full-sectioned samples, Mann-Whitney $\mathrm{U}$ test was performed to examine the difference in PCLB4 mRNA abundance between adjacent normal tissue and GISTs and between high-risk and non-highrisk groups. Pearson correlation analysis was used to evaluate the association between mRNA abundance and protein immunoexpression. In the TMA validation set, we evaluated the associations of PLCB4 copy number and PLCB4 immunoexpression with clinicopathological factors using the Chi-square and Wilcoxon rank-sum tests for categorical and continuous variables, respectively. Follow-up data were available for 350 cases as of April 2009 (median, 49.9 months; range, 1-247). The endpoint was disease-free survival (DFS), which would not be confounded by imatinib therapy for disseminated diseases. Based on previously prognostic correlations for DFS [21], genotypes of GISTs were dichotomized into the favorable and unfavorable groups. The former included (i) PDGFRA mutation involving exons 12 or 18 , (ii) 3' tandem insertion of KIT exon 11 with or without point mutation, and (iii) single point mutation of KIT exon 11 . The unfavorable genotypes comprised (i) Ala502-Tyr503 insertion of KIT exon 9, (ii) wild type for KIT, PDGFRA, and $B R A F$ genes without loss of SDHA and SDHB, and (iii) 5' deletion of KIT exon 11 with or without point mutation. We used the log-rank test to compare univariate prognostic analyses. In the multivariate analyses, significant prognosticators with univariate $\mathrm{p}<0.05$ were generally introduced, including either NIH scheme or NCCN guidelines, while size and mitosis, being component factors of risk stratification, were not incorporated in the multivariate comparisons.

\section{ACKNOWLEDGMENTS}

The authors thank Kaohsiung Chang Gung genomic (CMRPG880251) and tissue bank (CLRPG8B0033 and CLRPG8E0161) core laboratories for technical assistance and Shih-Chen Yu, MS for her excellent skill in tissue microarray-based immunostains.

\section{CONFLICTS OF INTEREST}

No conflicts of interest were declared.

\section{GRANT SUPPORT}

This work was sponsored by Taiwan National Science Council (NSC99-2628-B-182A-064-MY3, 102-2628-B-182A-002-MY3 to HYH, NSC99-2320- 
B-384-001-MY2 to CFL), Chang Gung Hospital (CMRPG8C0983 to HYH, CMRPG8C0991 to TTL) and Taiwan Ministry of Health and Welfare (MOHW103-TDU-M-221-123017 and MOHW104TDU-M-212-133004 to CFL) and Taiwan National Health Research Institutes (CA-106-PP-36 to CFL).

\section{Author contributions}

HYH, CFL, and TTL conceived experiments, analyzed data, and prepared the manuscript. CIC, YYC, and FMF collected and analyzed data. TTL, CIC, and TCC carried out the experiments. All authors were involved in writing the paper and had final approval of the submitted manuscript.

\section{REFERENCES}

1. Heinrich MC, Corless CL, Blanke CD, Demetri GD, Joensuu H, Roberts PJ, Eisenberg, BL, von Mehren M, Fletcher CD, Sandau K, McDougall K, Ou WB, Chen $\mathrm{CJ}$, et al. Molecular correlates of imatinib resistance in gastrointestinal stromal tumors. J Clin Oncol. 2006; 24: 4764-4774.

2. Fletcher CD, Berman JJ, Corless C, Gorstein F, Lasota J, Longley BJ, Miettinen M, O'Leary TJ, Remotti H, Rubin BP, Shmookler B, Sobin LH, Weiss SW. Diagnosis of gastrointestinal stromal tumors: A consensus approach. Hum Pathol. 2002; 33: 459-465.

3. Demetri GD, von Mehren M, Antonescu CR, DeMatteo RP, Ganjoo KN, Maki R, Pisters PW, Raut CP, Riedel RF, Schuetze S, Sundar HM, Trent JC, Wayne JD. NCCN Task Force report: update on the management of patients with gastrointestinal stromal tumors. J Natl Compr Canc Netw. 2010; 8 Suppl 2: S1-41; quiz S42-44.

4. Joensuu H, Rutkowski P, Nishida T, Steigen SE, Brabec P, Plank L, Nilsson B, Braconi C, Bordoni A, Magnusson MK, Sufliarsky J, Federico M, Jonasson JG, et al. KIT and PDGFRA mutations and the risk of GI stromal tumor recurrence. J Clin Oncol. 2015; 33: 634-642.

5. Dematteo RP, Ballman KV, Antonescu CR, Maki RG, Pisters PW, Demetri GD, Blackstein ME, Blanke CD, von Mehren M, Brennan MF, Patel S, McCarter MD, Polikoff JA, et al. Adjuvant imatinib mesylate after resection of localised, primary gastrointestinal stromal tumour: a randomised, double-blind, placebo-controlled trial. Lancet. 2009; 373: 1097-1104.

6. Debiec-Rychter M, Cools J, Dumez H, Sciot R, Stul M, Mentens N, Vranckx H, Wasag B, Prenen H, Roesel J, Hagemeijer A, Van Oosterom A, Marynen P. Mechanisms of resistance to imatinib mesylate in gastrointestinal stromal tumors and activity of the PKC412 inhibitor against imatinib-resistant mutants. Gastroenterology. 2005; 128: $270-279$.
7. Cairns RA, Harris IS, Mak TW. Regulation of cancer cell metabolism. Nat Rev Cancer. 2011; 11: 85-95.

8. Johansson P, Aoude LG, Wadt K, Glasson WJ, Warrier SK, Hewitt AW, Kiilgaard JF, Heegaard S, Isaacs T, Franchina M, Ingvar C, Vermeulen T, Whitehead KJ, et al. Deep sequencing of uveal melanoma identifies a recurrent mutation in PLCB4. Oncotarget. 2016; 7: 4624-4631. doi: 10.18632/oncotarget.6614.

9. Kakiuchi T, Takahara T, Kasugai Y, Arita K, Yoshida N, Karube K, Suguro M, Matsuo K, Nakanishi H, Kiyono T, Nakamura S, Osada H, Sekido Y, et al. Modeling mesothelioma utilizing human mesothelial cells reveals involvement of phospholipase-C beta 4 in YAP-active mesothelioma cell proliferation. Carcinogenesis. 2016; 37 , 1098-1109.

10. Shah US, Dhir R, Gollin SM, Chandran UR, Lewis D, Acquafondata M, Pflug B. Fatty acid synthase gene overexpression and copy number gain in prostate adenocarcinoma. Hum Pathol. 2006; 37: 401-409.

11. Waugh MG. Chromosomal Instability and Phosphoinositide Pathway Gene Signatures in Glioblastoma Multiforme. Mol Neurobiol. 2016; 53: 621-630.

12. Bunney TD, Katan M. Phosphoinositide signalling in cancer: beyond PI3K and PTEN. Nat Rev Cancer. 2010; 10: $342-352$.

13. Raimondi C, Falasca M. Phosphoinositides signalling in cancer: focus on PI3K and PLC. Adv Biol Regul. 2012; 52: 166-182.

14. Kunze K, Spieker T, Gamerdinger U, Nau K, Berger J, Dreyer T, Sindermann JR, Hoffmeier A, Gattenlohner S, Brauninger A. A recurrent activating PLCG1 mutation in cardiac angiosarcomas increases apoptosis resistance and invasiveness of endothelial cells. Cancer Res. 2014; 74 : 6173-6183.

15. Bertagnolo V, Benedusi M, Querzoli P, Pedriali M, Magri E, Brugnoli F, Capitani S. PLC-beta2 is highly expressed in breast cancer and is associated with a poor outcome: a study on tissue microarrays. Int J Oncol. 2006; 28: 863-872.

16. Bertagnolo V, Benedusi M, Brugnoli F, Lanuti P, Marchisio M, Querzoli P, Capitani S. Phospholipase C-beta 2 promotes mitosis and migration of human breast cancer-derived cells. Carcinogenesis. 2007; 28: 1638-1645.

17. Fukami K, Inanobe S, Kanemaru K, Nakamura Y. Phospholipase $\mathrm{C}$ is a key enzyme regulating intracellular calcium and modulating the phosphoinositide balance. Prog Lipid Res. 2010; 49: 429-437.

18. Gordon CT, Vuillot A, Marlin S, Gerkes E, Henderson A, AlKindy A, Holder-Espinasse M, Park SS, Omarjee A, Sanchis-Borja M, Bdira EB, Oufadem M, SikkemaRaddatz B, et al. Heterogeneity of mutational mechanisms and modes of inheritance in auriculocondylar syndrome. $\mathrm{J}$ Med Genet. 2013; 50: 174-186.

19. Cerami E, Gao J, Dogrusoz U, Gross BE, Sumer SO, Aksoy BA, Jacobsen A, Byrne CJ, Heuer ML, Larsson E, Antipin 
Y, Reva B, Goldberg AP, et al. The cBio cancer genomics portal: an open platform for exploring multidimensional cancer genomics data. Cancer Discov. 2012; 2: 401-404.

20. Hanna WM, Ruschoff J, Bilous M, Coudry RA, Dowsett M, Osamura RY, Penault-Llorca F, van de Vijver M, Viale G. HER2 in situ hybridization in breast cancer: clinical implications of polysomy 17 and genetic heterogeneity. Mod Pathol. 2014; 27: 4-18.

21. Li CF, Chen LT, Lan J, Chou FF, Lin CY, Chen YY, Chen TJ, Li SH, Yu SC, Fang FM, Tai HC, Huang HY. AMACR amplification and overexpression in primary imatinib-naive gastrointestinal stromal tumors: a driver of cell proliferation indicating adverse prognosis. Oncotarget. 2014; 5: 1158811603. doi: 10.18632/oncotarget. 2597.

22. Knudsen BS, Allen AN, McLerran DF, Vessella RL, Karademos J, Davies JE, Maqsodi B, McMaster GK, Kristal AR. Evaluation of the branched-chain DNA assay for measurement of RNA in formalin-fixed tissues. J Mol Diagn. 2008; 10: 169-176.

23. Wang YH, Wu WJ, Wang WJ, Huang HY, Li WM, Yeh BW, Wu TF, Shiue YL, Sheu JJ, Wang JM, Li CF. CEBPD amplification and overexpression in urothelial carcinoma: a driver of tumor metastasis indicating adverse prognosis. Oncotarget. 2015; 6:31069-84. doi: 10.18632/ oncotarget.5209.

24. Li CF, Wu WJ, Wu WR, Liao YJ, Chen LR, Huang CN, Li CC, Li WM, Huang HY, Chen YL, Liang SS, Chow NH, Shiue YL. The cAMP responsive element binding protein 1 transactivates epithelial membrane protein 2 , a potential tumor suppressor in the urinary bladder urothelial carcinoma. Oncotarget. 2015; 6:9220-39. doi: 10.18632/ oncotarget.3312.
25. Chi JY, Hsiao YW, Li CF, Lo YC, Lin ZY, Hong JY, Liu YM, Han X, Wang SM, Chen BK, Tsai KK, Wang JM. Targeting chemotherapy-induced PTX3 in tumor stroma to prevent the progression of drug-resistant cancers. Oncotarget. 2015; 6: 23987-4001. doi: 10.18632/ oncotarget.4364.

26. Li CF, Chuang IC, Liu TT, Chen KC, Chen YY, Fang FM, Li SH, Chen TJ, Yu SC, Lan J, Huang HY. Transcriptomic reappraisal identifies MGLL overexpression as an unfavorable prognosticator in primary gastrointestinal stromal tumors. Oncotarget. 2016; 7:49986-49997. doi: 10.18632/oncotarget.10304.

27. Ma LJ, Lee SW, Lin LC, Chen TJ, Chang IW, Hsu HP, Chang KY, Huang HY, Li CF. Fibronectin overexpression is associated with latent membrane protein 1 expression and has independent prognostic value for nasopharyngeal carcinoma. Tumour Biol. 2014; 35: 1703-1712.

28. Fukushima T, Suzuki S, Mashiko M, Ohtake T, Endo Y, Takebayashi Y, Sekikawa K, Hagiwara K, Takenoshita S. BRAF mutations in papillary carcinomas of the thyroid. Oncogene. 2003; 22: 6455-6457.

29. Rossi S, Sbaraglia M, Dell'Orto MC, Gasparotto D, Cacciatore M, Boscato E, Carraro V, Toffolatti L, Gallina G, Niero M, Pilozzi E, Mandolesi A, Sessa F, et al. Concomitant KIT/BRAF and PDGFRA/ BRAF mutations are rare events in gastrointestinal stromal tumors. Oncotarget. 2016; 7:30109-18. doi: 10.18632/ oncotarget.8768.

30. Jasek K, Buzalkova V, Minarik G, Stanclova A, Szepe P, Plank L, Lasabova Z. Detection of mutations in the BRAF gene in patients with KIT and PDGFRA wildtype gastrointestinal stromal tumors. Virchows Arch. 2016 doi:10.1007/s00428-016-2044-4. 